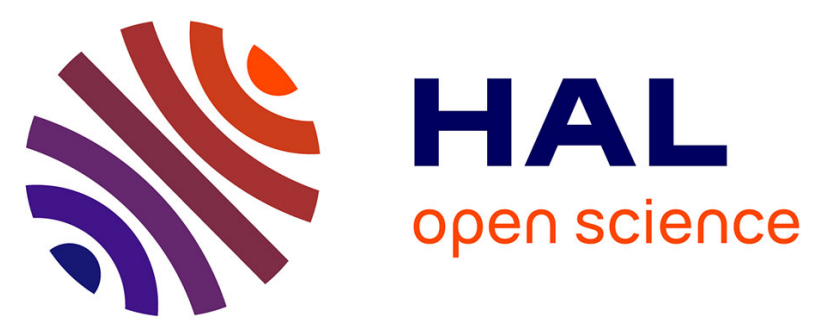

\title{
Gate defects analysis in AlGaN/GaN devices by mean of accurate extraction of the Schottky Barrier Height, electrical modelling, T-CAD simulations and TEM imaging
}

Jean-Guy Tartarin, Oana Lazar, Damien Saugnon, B. Lambert, C. Moreau, C. Bouexiere, E. Romain-Latu, K. Rousseau, A. David, J.L. L Roux

\section{To cite this version:}

Jean-Guy Tartarin, Oana Lazar, Damien Saugnon, B. Lambert, C. Moreau, et al.. Gate defects analysis in AlGaN/GaN devices by mean of accurate extraction of the Schottky Barrier Height, electrical modelling, T-CAD simulations and TEM imaging. Microelectronics Reliability, 2017, 76-77, pp.344-349. 10.1016/j.microrel.2017.07.057 . hal-02088137

\section{HAL Id: hal-02088137 \\ https://hal.laas.fr/hal-02088137}

Submitted on 2 Apr 2019

HAL is a multi-disciplinary open access archive for the deposit and dissemination of scientific research documents, whether they are published or not. The documents may come from teaching and research institutions in France or abroad, or from public or private research centers.
L'archive ouverte pluridisciplinaire HAL, est destinée au dépôt et à la diffusion de documents scientifiques de niveau recherche, publiés ou non, émanant des établissements d'enseignement et de recherche français ou étrangers, des laboratoires publics ou privés. 


\title{
Gate Defects Analysis in AlGaN/GaN Devices by Mean of Accurate Extraction of the Schottky Barrier Height, Electrical Modeling, T-CAD Simulations and TEM imaging
}

\author{
J.G. Tartarinn,"*, O. Lazar ${ }^{\mathrm{a}}$, D. Saugnon ${ }^{\mathrm{a}}$, B. Lambert ${ }^{\mathrm{b}}$, C. Moreau ${ }^{\mathrm{c}}$, C. Bouexiere ${ }^{\mathrm{c}}$,

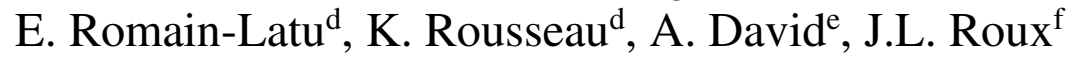 \\ a LAAS-CNRS and Université de Toulouse, Université Paul Sabatier, 31031 Toulouse, France \\ ${ }^{\mathrm{b}}$ United Monolithic Semiconductors, Villebon-sur Yvette, 91140, France \\ ' DGA MI, Rennes, 35998, France \\ d SERMA Technologies MINATEC BHT, Grenoble, 38040, France \\ ${ }^{\mathrm{e}}$ Biophy Research, Fuveau,13710, France \\ ${ }^{\mathrm{f}}$ CNES, Quality Assurance Directorate Department, Toulouse, 31400, France
}

\begin{abstract}
This paper proposes an investigation focused on the Schottky Diode related electrical behaviors on GaN high frequency technologies. As the Schottky Diode represents the electrical input terminal (the command) of High Electron Mobility Transistors (HEMTs), this study also correlates with some first order degradation in the active channel (current $\mathrm{I}_{\mathrm{DS}}$ ). Non-invasive methods and related models have been used to determine the accurate Schottky Barrier Height $(\mathrm{SBH})$ of the diode in terms of mean value and dispersion; this approach is convenient to evidence different failure mechanisms on virgin and stressed devices that can be correlated with DC or transient electrical parameters. It is shown that according to given temperature windows and $\mathrm{I}_{\mathrm{GS}}$ ranges, linear relationships can be extracted between the mean SBH and the inhomogeneities of the SBH that appear in forward-biased diode mode. This original approach permits to determine an increase or a decrease of the global SBH after a stress period. Electrical behaviors issued from the proposed non-destructive technique and from electrical modelling of the diode at different temperatures are found to be consistent with Transmission Electron Microscope (TEM) investigations. TCAD models have also been used and tuned to account for the impact of interface fixed charge density changes on the electrical signatures of the HEMTs.
\end{abstract}

\section{Corresponding author.}

tartarin@laas.fr Tel: +33(0)56133 7996; Fax: +33 (0) 561336300 


\title{
Gate Defects Analysis in AlGaN/GaN Devices by Mean of Accurate Extraction of the Schottky Barrier Height, Electrical Modeling, T-CAD Simulations and TEM Imaging
}

\author{
J.G. Tartarin a,*, O. Lazar ${ }^{\mathrm{a}}$, D. Saugnon ${ }^{\mathrm{a}}$, B. Lambert ${ }^{\mathrm{b}}$, C. Moreau ${ }^{\mathrm{c}}$, C. Bouexiere ${ }^{\mathrm{c}}$, \\ E. Romain-Latu ${ }^{\mathrm{d}}$, K. Rousseau ${ }^{\mathrm{d}}$, A. David ${ }^{\mathrm{e}}$, J.L. Roux ${ }^{\mathrm{f}}$
}

\section{Introduction}

ALGAN/GAN HEMTs are already known for their outstanding performances in microwave field of applications. The high breakdown voltage, electron saturation velocity, cutoff frequency and figures of merit, reveal them as attractive competitors towards the classical solid state transistors developed on silicon and GaAs materials. Using $\mathrm{GaN}$ for electronic circuits results in a loss in weight for the system (reduction in the electronic circuit area, power supply and heater or cooling system sizes), with enhanced power capabilities. Yet, limitations like the increase of the junction temperature still represent a challenge to broaden the application fields and markets. Systems working at elevated junction temperature can open the way for new high power and high frequency applications, as for example large volume production dedicated to base stations and other high volume markets, but also to defense and space purposes working under harsh environment. However, reliability investigations are still largely under concern because of the complex electro-thermo-mechanical mechanisms induced in transistors; these reliability studies can only be carried out by using several cross-experiments, or by using destructive techniques in order to evidence the presence of defects. Nevertheless, destructive techniques do not allow concluding about the real impact of the revealed defect on the electrical behavior of the device. The development of new non-invasive (and then non-destructive) experimental approaches can thus bring information about the evolution of electrical signatures before (during) and after application of a stress. This study specifically focuses on gate barrier height related defects, as it impacts the control of the charges in the channel where the electron carriers flow, and interface states causing various reliability issues. Devices were tested under High Temperature Operating Life stress conditions (HTOL). T-CAD model is also developed to account for the fine technological mechanism analysis of the gate control zone, as well as interface fixed charge densities effects on static electrical parameters.

This document is structured as follows: the second section briefly describes the technology under test and the stress conditions used on a sample of 10 devices. The third section is dedicated to the extraction method of the Schottky Barrier Height (SBH) and associated electrical models as well as T-CAD simulations. The last section deals with TEM pictures at the gate edges and under the gate. Lastly, a conclusion is drawn which states the usefulness of the proposed method in terms of technological feedback.

\section{Technology under test and under stress}

Results on an intermediate step of the final technological declination (under development phase) are reported, with the aim to assess the potential of our method. A batch of ten AlGaN/GaN HEMT devices featuring eight gate fingers, with a width of $125 \mu \mathrm{m}$ each (technology $0.25 \mu \mathrm{m}$ gate length), grown on $\mathrm{SiC}$ substrate have been used for the study. Two virgin devices are available to evaluate the impact of the HTOL stress operated by UMS at $\mathrm{V}_{\mathrm{DS}}=30 \mathrm{~V}$ and $\mathrm{I}_{\mathrm{DS}}=100 \mathrm{~mA} / \mathrm{mm}$, at $175^{\circ} \mathrm{C}$ ambient temperature $\left(320^{\circ} \mathrm{C}\right.$ peak junction temperature extracted from simulation model). The measurements reveal a drop on $\mathrm{I}_{\mathrm{DS}}$ of 
about $45 \%$ (at $V_{G S}=1 \mathrm{~V}$ and $V_{D S}=8 \mathrm{~V}$ ), an increase of $130 \%$ on $R_{O N}$, and about one decade variation on $I_{G S}$ after 105 hours of HTOL stress. Threshold voltage relative evolutions during the stress are reported in Fig. 1, with final shifts between $5 \%$ and $15 \%$ on stressed devices (no variation on the two virgin devices also measured - black and red plots).

The demand of having a clean gate contact in power devices is still a key challenge in achieving reliable processes, since some of the failure mechanisms are related to the specific development of the Schottky gate contact. Moreover, the gate terminal (i.e., the command) is the critical zone impacting the performances of the device: shift of the threshold voltage $V_{t h}$, leads to variation of the transconductance gain and of the carriers' density in the two-dimensional electron gas, which become detrimental to the circuit operation and to the system performances.

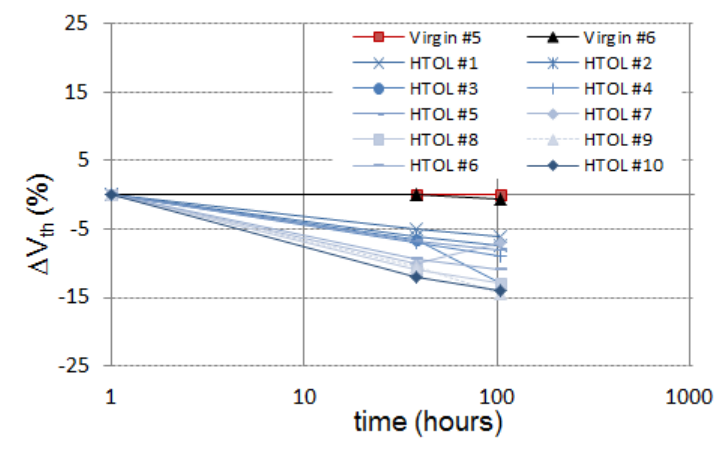

Fig. 1. Impact of the HTOL stress on threshold voltage shift (expressed in percentage).

\section{Electrical measurements and modelling of the Gate Schottky Diode}

From previous studies [1]-[3], different methods have been developed to investigate the gate contact. Since we are talking about diodes and non-destructive techniques, the most common approach consists in the analysis of the SBH. This part of the work is based on prior studies [4][5], which present an accurate tool for extracting the SBH alone, through the quantification of the defects impact on the global SBH. The technique from [5] brings information about a mean $\mathrm{SBH} \overline{\Phi_{B x}}$ and its relative dispersion $\sigma_{S x}$ (inhomogeneity of the barrier, Fig. 2). This technique is non-invasive as it is based on electro-thermal characterization of the device, in open drain configuration (no dissipated power in the

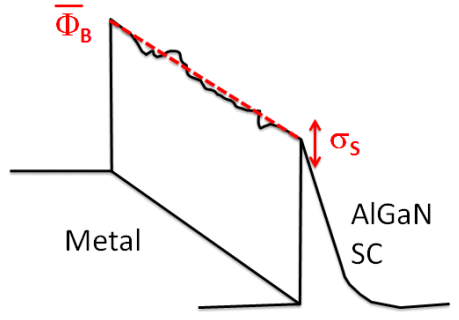

Fig. 2. Representation of the parameters from Werner's method applied to Richardson's model as expressed in [5]: a constant mean value of the $\mathrm{SBH}\left(\overline{\Phi_{B x}}\right)$ and an associated standard deviation $\left( \pm \sigma_{S x}\right)$, i.e. a mathematical expression for the SBH's inhomogeneity.

2DEG). Fig. 3 plots the gate current versus the gate voltage for a virgin device $\mathrm{V}_{\# 6}$ (Fig. 3.a) and for a stressed device $S_{\# 2}$ (Fig. 3.b) at different temperatures. Fig. 4 gives the thermal evolution of the SBH expressed according to its classical electrical expression. Two thermal trends from part of a thermal inflexion point are observed.

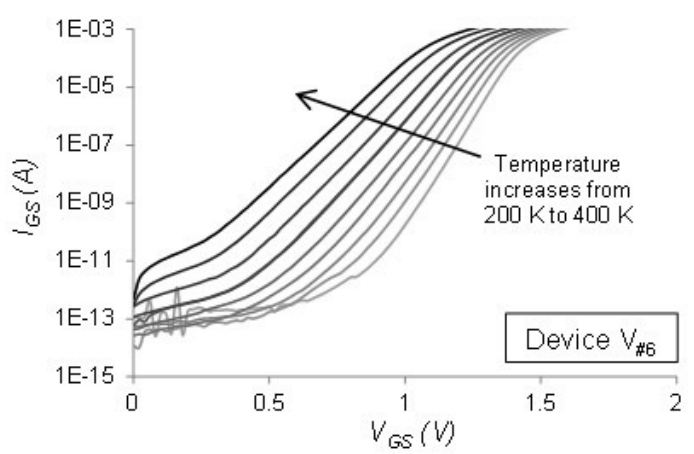

a)

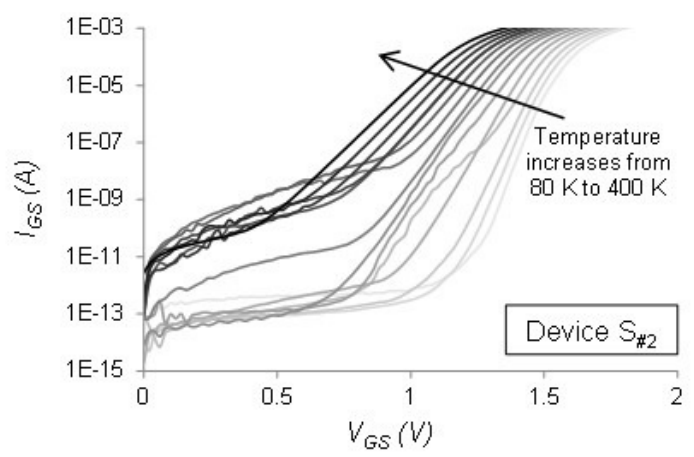

b)

Fig. 3. $I_{G S}-V_{G S}$ measurements versus temperature, with a $25 \mathrm{~K}$ step for: a) $\mathrm{V}_{\# 6}$ (virgin sample) and b) $\mathrm{S}_{\# 2}$ (stressed sample). Light grey is represented as the lowest temperature, towards dark black as the highest temperature. 


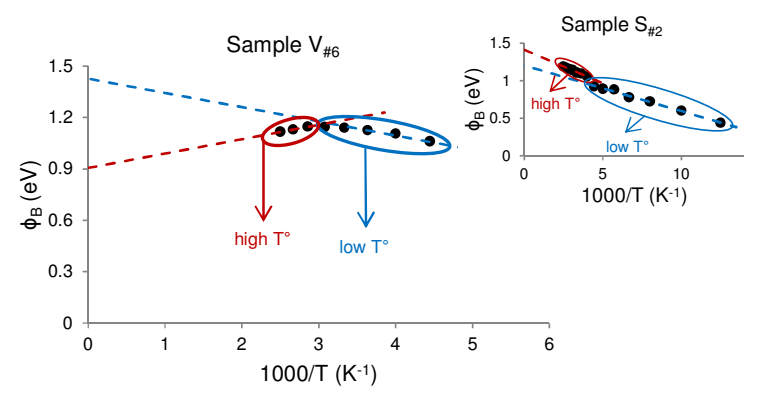

Fig. 4. SBH extracted from classical expression versus temperature for a virgin device $\mathrm{V}_{\# 6}$ and for a stressed device $S_{\# 2}$. An inflexion point separates two temperature zones, next called high $\mathrm{T}^{\circ}$ (red) and low $\mathrm{T}^{\circ}$ (blue).

3.1. Extraction of the mean SBH and dispersionextracting the accurate ideal $S B H$

From [5], the determination of the mean SBH and a related dispersion $\left(\overline{\Phi_{B x}}, \sigma_{S x}\right)$ is found not to be sufficient to get the Schottky Barrier Height determined accurately. By varying the $\mathrm{I}_{\mathrm{GS}}$ current range (considering a 2-decades window) or by changing the temperature, it is demonstrated that:

-the mean SBH evolves largely out of the uncertainty zone of the method, and depends on the $\mathrm{I}_{\mathrm{GS}}$ range,

-the trend of the mean SBH also depends on the temperature window (next called High Temperature and Low Temperature zones) as depicted in Fig. 4.

Concerning the extraction's accuracy of the mean SBH associated to the method proposed in [5], it has been established that:

-errors due to signal analyzer measurements can be considered insignificant

-an error of less than $7 \%$ on $\overline{\Phi_{B x}}$ is obtained for $50 \%$

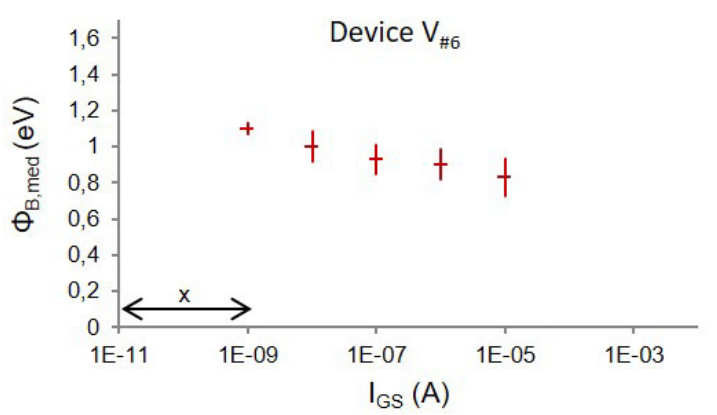

Fig. 5. Example for the extracted mean $\overline{\Phi_{B x}}$ against the ' $x$ ' sliding window ( 2 decades $\Delta \mathrm{I}_{\mathrm{GS}}$ ), considering the variation of the standard deviation $\sigma_{S x}$, vertical error bars (illustration on virgin device \#6). variation on prerequisite initial conditions on Richardson constant $\mathrm{A}^{*}$ and effective mass $\mathrm{m}^{*}$.

-the larger variation is noticed when considering the standard deviation associated to the extracted SBH $\overline{\Phi_{B x}}$ for each ' $x$ ' sliding window of $\Delta \mathrm{I}_{\mathrm{GS}}$ (Fig. 5). From a dedicated study, it has been established that two decades window for $\Delta \mathrm{I}_{\mathrm{GS}}$ features the lowest variations.

An original reinterpretation of the pairs $\left(\overline{\Phi_{B x}}, \sigma_{S x}\right)$ has been proposed in [5] by plotting $\overline{\Phi_{B x}}$ versus $\sigma_{S x}$ for all the ' $x$ ' sliding windows of two decades of $\Delta I_{G S}$ (Fig. $6 \mathrm{a}$ and $\mathrm{b}$ ). Therefore, the variation of the mean SBH versus the standard deviation (inhomogeneity) represents the sensitivity of the ideal SBH's value to the defects of the barrier height (inhomogeneity at the metal-semiconductor interface). Thus, the $x$-axis extrapolation at $\sigma_{\mathrm{S}}=0 \mathrm{eV}$ represents the ideal Schottky diode without considering any defect $\left(\Phi_{\mathrm{B}-\sigma_{\mathrm{s}}=0}\right.$; expected theoretical homogeneous SBH).

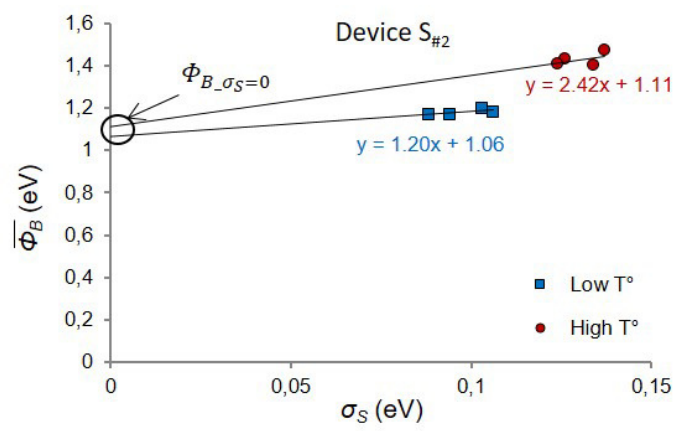

a)

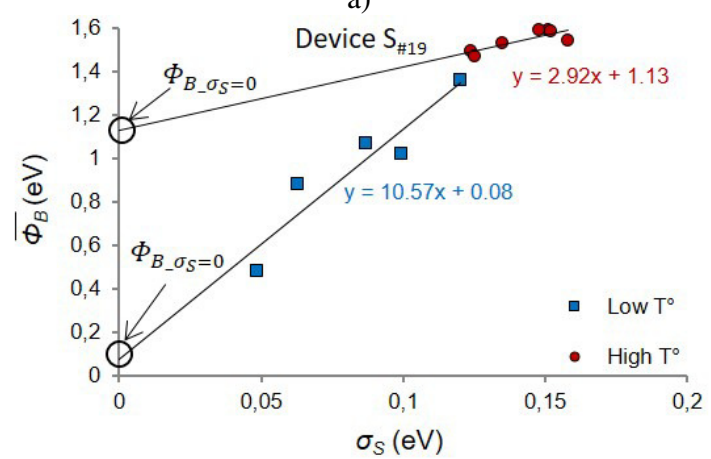

b)

Fig. 6. Variations of $\overline{\Phi_{B x}}$ versus $\sigma_{S x}$ under different gate current ranges (x-sliding gate current window), for: a) $\mathrm{S}_{\# 2}$ (stressed device \#2), and b) $S_{\# 19}$ (stressed sample \#19). Squares represent the pairs $\left(\overline{\Phi_{B x}}, \sigma_{S x}\right)$ in the low temperature range and circles are the pairs $\left(\overline{\Phi_{B x}}, \sigma_{S x}\right)$ in the high temperature range. 


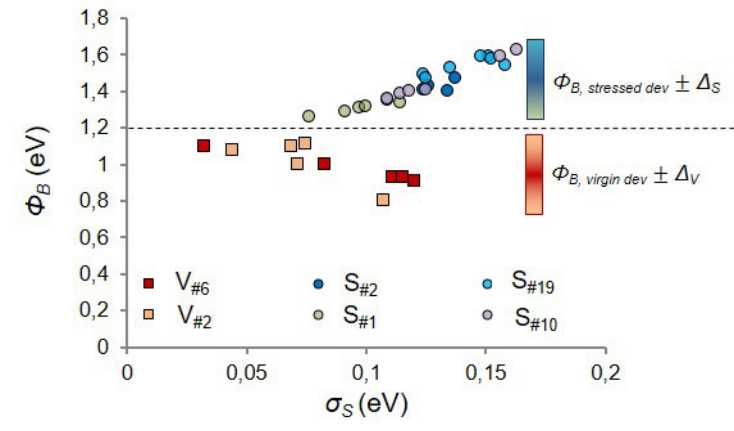

Fig. 7. Variations of $\overline{\Phi_{B x}}$ versus $\sigma_{S x}$ at high temperature, under different gate current ranges (x-sliding gate current window), for 6 devices: (2 virgin samples V\# squares -4 stressed samples S\# circles)

The different trends revealed at low or high temperature in Fig. 6.a and Fig. 6.b are consistent with first order and second order defects of the Schottky diode: however, whatever the increasing (stressed devices) or decreasing (virgin devices) impact of the defect within the high temperature window (see Fig. 7), a unique $\Phi_{\mathrm{B}-\sigma_{\mathrm{s}=0}}$ is extracted at $1.1 \mathrm{eV}( \pm 0.1 \mathrm{eV})$. For the lower temperature range, some devices still exhibit an extrapolation of $\Phi_{\mathrm{B}-\sigma_{\mathrm{s}}=0}$ at $1.1 \mathrm{eV}$ while two devices give an extrapolation at $0 \mathrm{eV}$ (see Fig. 6.b, blue squares). This behavior correlates with Energy Dispersive X-Ray spectrometry (EDX) measurements, evidencing a gold conduction path at the edge of the gate as previously shown in [6].

\subsection{Electrical models of the gate diode versus} temperature

Electrical model has been developed and tuned for all temperatures under study and for all devices under

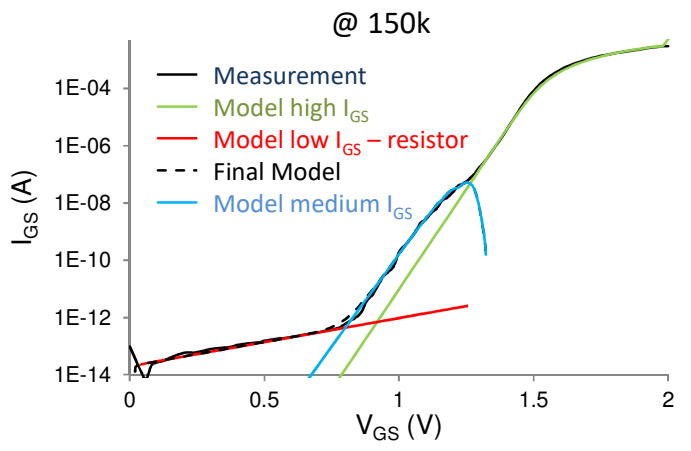

Fig. 8. Example of a model for the gate Schottky diode for a temperature of $150 \mathrm{~K}$, featuring different contributions to $\mathrm{I}_{\mathrm{GS}}$ at low, medium or high $\mathrm{I}_{\mathrm{GS}}$ levels. test, revealing different zones in forward biasing conditions that correlate the results from the previous section (TE mode). Fig. 8 reports $\mathrm{I}_{\mathrm{GS}}-\mathrm{V}_{\mathrm{GS}}$ plot at $150 \mathrm{~K}$ where a second diode is identified at medium $\mathrm{I}_{\mathrm{GS}}$, but with higher serial resistor (revealing a conduction path not flowing into the quantum well). A highly resistive behaviour is also revealed (ranging from $10^{8}$ to $10^{12} \Omega$ ) at low $\mathrm{I}_{\mathrm{GS}}$ attributed to leakage current at the interface between the SiN passivation and the AlGaN layer (no Schottky diode is involved in this electrical path). This resistive behaviour is reported for 5 devices under test in Fig. 9 ( 4 devices featuring $4.10^{9} \Omega$ as illustrated in the inset, and 1 virgin device featuring a higher $10^{12} \Omega$ resistance). The measurements are reproducible.

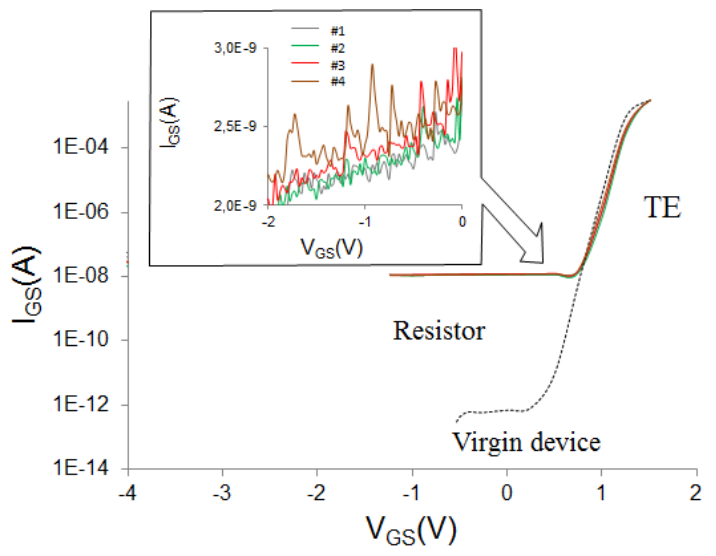

Fig. 9. Extraction of the resistive parasitic behavior from $\mathrm{I}_{\mathrm{GS}}-\mathrm{V}_{\mathrm{GS}}$ plots in diode mode $\left(\mathrm{V}_{\mathrm{DS}}=0 \mathrm{~V}\right)$ over a batch of 5 samples (4 devices featuring a $4.10^{9} \Omega$ resistance, 1 virgin device feat. $10^{12} \Omega$ resistance), with Thermionic Emission (TE) mode used for SBH extraction.

\subsection{T-CAD models}

T-CAD simulator (Sentaurus) has been instructed and tuned to match both the stressed and virgin devices on output characteristics $\mathrm{I}_{\mathrm{DS}}-\mathrm{V}_{\mathrm{DS}}$ and transfer characteristics $\mathrm{I}_{\mathrm{DS}}-\mathrm{V}_{\mathrm{GS}}\left(\mathrm{G}_{\mathrm{m}}-\mathrm{V}_{\mathrm{GS}}\right)$. The implementation of the modified structure for the stressed devices is realized considering DC (and transient) electrical measurements performed on $\mathrm{I}_{\mathrm{GS}}$ and $\mathrm{I}_{\mathrm{DS}}$. The principal focus of this section concerns the impact of fixed charges (FC) at the different interfaces from the surface to the active 2DEG, under the gate and between gatesource and gate-drain zones as depicted in Fig. 10.

The different zones where the fixed charges are considered are the gated zone (\#A under the gate finger) and the ungated zones (\#B between gate and source, or gate and drain). Then, simulations are 

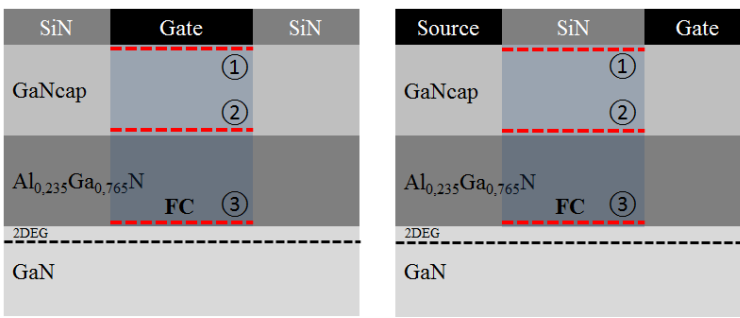

\#A: under the gate finger

Fig. 10. HEMT cut view pattern for Fixed Charges (FC) density impact evaluation on electrical behaviors before and after stress application.

performed at three different interfaces: (1) FC under the gate metal (\#A) or passivation layer (\#B), (2) at the GaNcap/AlGaN interface and (3) at the AlGaN/GaN (2DEG) interface. These FC locations at (1), (2) and (3) are considered independently and simultaneously. Only the FC density at the $\mathrm{AlGaN} / \mathrm{GaN}$ interface (3) is found to be critical to the variations of $I_{D S S}, R_{O N}$ and $\mathrm{V}_{\text {th }}$ (weak contribution of FC located at (2) and no impact of $\mathrm{FC}$ at (1)). Fig. 11 reports on the intrinsic transconductance gain $\mathrm{g}_{\mathrm{m}}$ variation for different FC densities as depicted in \#A, under the gate finger. Only the threshold voltage $\mathrm{V}_{\text {th }}$ shifts when FC density varies in this case. When considering $\mathrm{FC}$ in the ungated zones of the HEMT (i.e. G-S or G-D zones), then IDss (and $R_{O N}$ ) changes as reported on $g_{m}$ profiles versus $V_{G S}$ in Fig. 12, whereas $\mathrm{V}_{\text {th }}$ remains constant. $\mathrm{T}$-CAD simulations enable the FC effects at the interfaces of the \#A gated zone to be differentiated from that in the

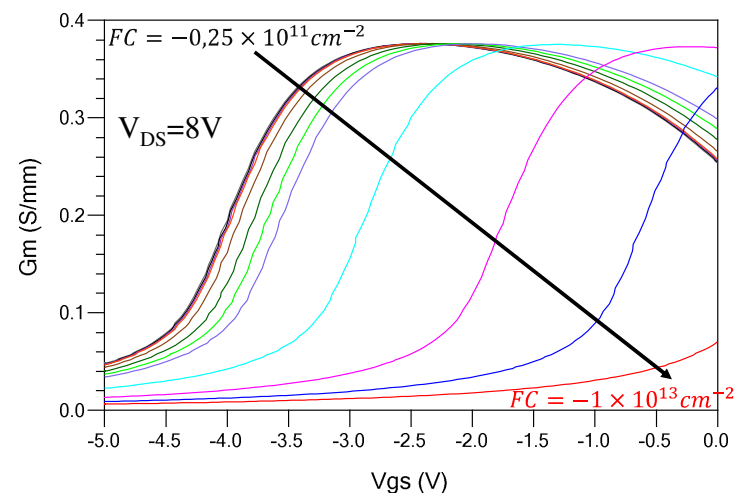

Fig. 11. Simulated transconductance gain with $V_{D S}=8 V$ for different fixed charges densities FC under the gate finger (case \#A, negative FC at interface (3)). FC ranges from $-0,25.10^{11} \mathrm{~cm}^{-2}$ to $-1.10^{11} \mathrm{~cm}^{-2}$ (step $0,25.10^{11} \mathrm{~cm}^{-2}$ ), from $-0,25.10^{12} \mathrm{~cm}^{-2}$ to $-1.10^{12} \mathrm{~cm}^{-2}$ (step $0,25.10^{12} \mathrm{~cm}^{-2}$ ) and from $-0,25 \cdot 10^{13} \mathrm{~cm}^{-2}$ to $-1 \cdot 10^{13} \mathrm{~cm}^{-2}$ (step $0,25 \cdot 10^{13} \mathrm{~cm}^{-2}$ ).

\#B ungated zones over the electrical behaviours before and after application of the stress.

The average $35 \%$ reduction on $\mathrm{IDSS}_{\mathrm{DS}}$ is then achieved by simulation using a higher FC density in the ungated zone \#B (increase by $\triangle \mathrm{FC}=-1,2 \cdot 10^{12} \mathrm{~cm}^{-2}$ ). The sensitivity of $\mathrm{V}_{\text {th }}$ with fixed charge variation $(\triangle \mathrm{FC})$ is constant whatever the initial $\mathrm{FC}$ density, and equal to $\Delta \mathrm{V}_{\mathrm{th}} / \Delta \mathrm{FC}=0.5 .10^{-12} \mathrm{~V} / \mathrm{cm}^{-2}$. Then, simulations corroborate well with the measurements (featuring $+0.2 \mathrm{~V}$ to $+0.5 \mathrm{~V}$ increase in $\mathrm{V}_{\text {th }}$ after HTOL stress, i.e. $6 \%$ to $15 \%$ in Fig. 1), if using a fixed charge density variation $\triangle \mathrm{FC}$ between $0,4.10^{12} \mathrm{~cm}^{-2}$ and $10^{12} \mathrm{~cm}^{-2}$. It is also noticed a positive shift of $\mathrm{V}_{\text {th }}$ when performing transient measurements as evidenced in [7]. Different evolutions can be found for $\mathrm{V}_{\text {th }}$ in the literature: a drift towards more negative threshold values after stress periods is reported in [8], caused by substitutional and interstitial oxygen complexes but featuring recoverable effects due to the nature of these defects.

Two simulation plans are used as depicted in Fig. 10 ( \#A and \#B); an average of $\Delta \mathrm{FC}=10^{12} \mathrm{~cm}^{-2}$ variation in the density of fixed charge at the AlGaN/2DEG interface accounts both for the $V_{\text {th }}$ shift and for the IDSs reduction (first order G-S spacing and second order G-D spacing). Fixed Charges variation between gate and drain account for the $\mathrm{R}_{\mathrm{ON}}$ increase. These results are consistent with some other papers, even if related to recoverable effects [10].

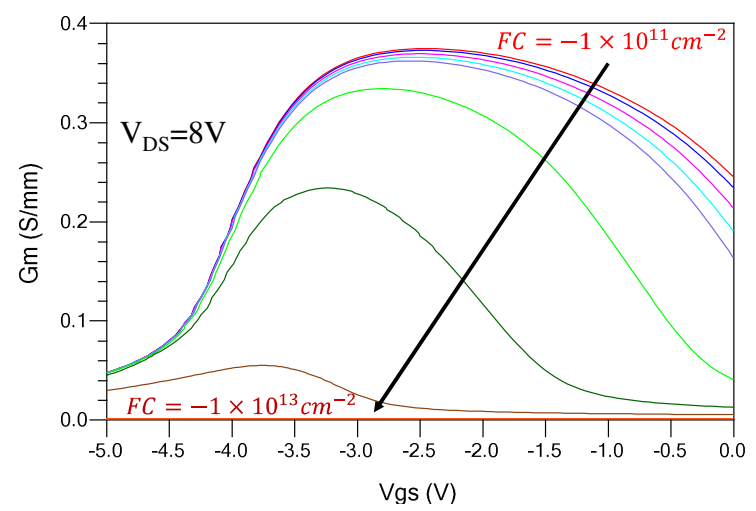

Fig. 12. Simulated transconductance gain with $\mathrm{V}_{D S}=8 \mathrm{~V}$ for different fixed charges densities FC under the ungate G-S (GD) zone(s) (case \#B, negative $\mathrm{FC}$ at interface (3)). FC ranges from $-0,25.10^{11} \mathrm{~cm}^{-2}$ to $-1.10^{11} \mathrm{~cm}^{-2}$ (step $0,25.10^{11} \mathrm{~cm}^{-2}$ ), from $-0,25.10^{12} \mathrm{~cm}^{-2}$ to $-1.10^{12} \mathrm{~cm}^{-2}$ (step $0,25.10^{12} \mathrm{~cm}^{-2}$ ) and from $-0,25.10^{13} \mathrm{~cm}^{-2}$ to $-1.10^{13} \mathrm{~cm}^{-2}$ (step $0,25.10^{13} \mathrm{~cm}^{-2}$ ) 


\section{Imaging with STEM HAADF and TEM lamella}

TEM analysis gives a direct proof of structural defects in GaN structures, and has led to major issues for $\mathrm{GaN}$ technology improvements from different works, as in [9]. Next studies on STEM HAADF and TEM lamella have been driven by DGA MI (French MoD) on HTOL stressed devices.

\subsection{Scanning Transmission Electron Microscopy} High Angle Annular Dark Field (STEM-HAADF) section view

STEM-HAADF images are suitable to define clearly the interface between $\mathrm{GaN}, \mathrm{AlGaN}, \mathrm{SiN}$ passivation layer or Gate Schottky metal; a FIB cut in the gate region allows an imaging of the stacked layers. Strong contrast can be identified between the layers as proposed in Fig. $13 \mathrm{a}$ and $\mathrm{b}$ (respectively in the gatepassivation zone and under the gate metal). Contrasted domains are noticed at the interface between the passivation layer and the AlGaN layer. This 'unexpected' layer is constituted with $\mathrm{Ga}, \mathrm{Al}$ and $\mathrm{O}$ species (by EDX and EFTEM), and is considered to be resistive. This observation is consistent with the highly resistive behavior at low $\mathrm{I}_{\mathrm{GS}}$ (Fig. 8 and Fig. 9 inset).

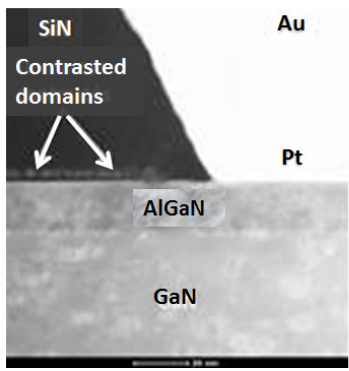

a) gate-source zone

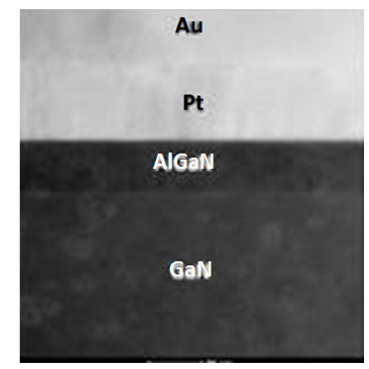

b) under the gate zone
Fig. 13. STEM-HAADF images (FIB cut) performed by Biophy Research a) on the gate edge between gate and source and b) under the gate.

4.2. TEM lamella by removing the gate metallization - top view

TEM lamella of the gate foot (top view after removing the metallization of the gate finger) is reported in Fig. 14; the presence of voids under the gate finger is consistent with the inhomogeneity of the SBH revealed in Fig. 6 and Fig. 7.

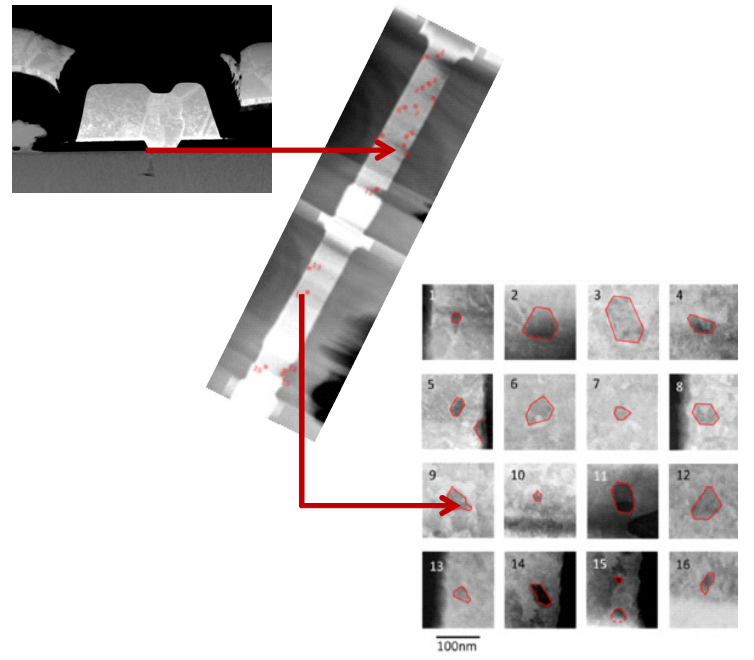

Fig. 14. TEM lamella of the gate finger (top view) performed by SERMA: identification of defects (voids) under the gate.

\section{Conclusions}

The identification of the impacting physical mechanism on the degradation of a (batch of) device(s) during the application of a stress is difficult to achieve. Usually, different contributors have to be considered together, but are not easily identifiable using a single method (experimental or simulation). Crossing dedicated measurements techniques and models with T-CAD simulations enlarges the field of investigation for failure analysis and process improvement. A failure analysis study case focused on gate Schottky diode inhomogeneity and interface states in the gated and ungated zones of the transistor have been presented.

It has been demonstrated the difficulty to achieve accurate Schottky Barrier Height extraction; the new proposed method accounts for the ideal SBH (extracted at $1.1 \mathrm{eV}$ ), and also evidence different scattering processes on the SBH before and after the application of the HTOL stress.

From an electrical model developed at different temperatures, several gate current contributors have been extracted (evidencing two SBH paths); a focus was given on a highly resistive behavior occurring near the Thermionic Emission mode.

These results on the SBH inhomogeneity and on the highly resistive layer at the passivation/AlGaN interface corroborate with the different TEM analysis of the gate proposed in section view and top view.

T-CAD model has also been developed to assess the HTOL stress induced variations on $\mathrm{I}_{\mathrm{DSs}}$ and on $\mathrm{V}_{\text {th. }}$. 
Fixed charge density variations at the AlGaN/2DEG interface have been used in the gated zone and in the ungated zones of the modeled transistor to account respectively for the $\mathrm{V}_{\text {th }}$ drift and for the $\mathrm{I}_{\mathrm{DSs}}$ reduction. Results also corroborate well with measurement considering the impact of $\Delta \mathrm{FC}=10^{12} \mathrm{~cm}^{-2}$ on $\mathrm{V}_{\text {th }}$ and on IDSs after combining the effects from each zone.

\section{References}

[1]J. H. Werner, H. H. Güttler, "Barrier inhomogeneities at Schottky contacts," J. Appl. Phys., vol. 69, no. 3, pp. 1522-1533, 1991.

[2]R. T. Tung, "Electron transport at metal-semiconductor interfaces: General theory," Phys. Rev. B, vol. 45, no. 23, pp. 13509-13523, 1992.

[3]S. Chand and J. Kumar, "Evidence for the double distribution of barrier heights in Pd2Si/n-Si Schottky diodes from I-V-T measurements," Semicond. Sci. Technol., vol. 11, no. 8, pp. 1203-1208, 1996.

[4] S. Karboyan et al. "Analysis of barrier inhomogeneities in AlGaN/GaN HEMTs' Schottky diodes by IVT measurements," Eur. Microw. Integr. Circuits Conf. EuMIC, pp. 240-243, 2013.

[5]O. Lazăr et al."New Approach for an Accurate Schottky Barrier Height's Extraction by IVT Measurements," IEEE Int. Microw. Symp. IMS, pp. 309-312, 2015.

[6] R. Stevenson, "Understanding Imperfections in $\mathrm{GaN}$ HEMTs" presented at CS Mantech, 2014.

[7] D. Bisi et al."Deep-level characterization in GaN HEMTs - Part I : Adv. and limitations of Drain current transient measurements", IEEE Trans. On Electron Devices, Vol. 60, No. 10, Oct. 2013, pp.3166-3175.

[8]D.M. Fleetwood et al."Oxygen-related border traps in MOS and GaN devices" IEEE $11^{\text {th }}$ Int. Conf. on SolidState and Integr. Circuit Techn. (ICSICT), 2012, 7p.

[9]S.Y. Park, "Physical degradation of GaN HEMT devices under high drain bias reliability testing", Microelectronics Reliability 49 (2009) 478-483.

[10] M. Meneghini et al. "Negative Bias-Induced Threshold Voltage Instability in GaN-on-Si Power HEMTs", IEEE EDL, Vol. 37, Issue 4 (2016) 474-477. 